\title{
In Vitro Construction of Scaffold-Free Bilayered Tissue-Engineered Skin Containing Capillary Networks
}

\author{
Yuan Liu, ${ }^{1}$ Hailang Luo, ${ }^{1,2}$ Xinwen Wang, ${ }^{3}$ Akimichi Takemura, ${ }^{4}$ Yi Ru Fang, \\ Yan Jin, ${ }^{1}$ and Fumihiko Suwa ${ }^{4}$ \\ ${ }^{1}$ Department of Oral Histology and Pathology, School of Stomatology, The Fourth Military Medical University, Xian 710032, China \\ ${ }^{2}$ Xi'an Institute of Tissue Engineering \& Regenerative Medicine, Xi'an 710032, China \\ ${ }^{3}$ Department of Periodontology \& Oral Medicine, School of Stomatology, The Fourth Military Medical University, Xi'an 710032, China \\ ${ }^{4}$ Department of Anatomy, Osaka Dental University, 8-1 Kuzuhahanazono-cho, Hirakata-shi, Osaka 573-1121, Japan \\ ${ }^{5}$ Department of Dental Oriental Medicine, Osaka Dental University, 8-1 Kuzuhahanazono-cho, Hirakata-shi, Osaka 573-1121, Japan
}

Correspondence should be addressed to Yan Jin; yanjin@fmmu.edu.cn and Fumihiko Suwa; suwa@cc.osaka-dent.ac.jp

Received 5 January 2013; Revised 21 February 2013; Accepted 22 February 2013

Academic Editor: Richard Tucker

Copyright (C) 2013 Yuan Liu et al. This is an open access article distributed under the Creative Commons Attribution License, which permits unrestricted use, distribution, and reproduction in any medium, provided the original work is properly cited.

\begin{abstract}
Many types of skin substitutes have been constructed using exogenous materials. Angiogenesis is an important factor for tissueengineered skin constructs. In this study, we constructed a scaffold-free bilayered tissue-engineered skin containing a capillary network. First, we cocultured dermal fibroblasts with dermal microvascular endothelial cells at a ratio of $2: 1$. A fibrous sheet was formed by the interactions between the fibroblasts and the endothelial cells, and capillary-like structures were observed after 20 days of coculture. Epithelial cells were then seeded on the fibrous sheet to assemble the bilayered tissue. HE staining showed that tissueengineered skin exhibited a stratified epidermis after 7 days. Immunostaining showed that the epithelium promoted the formation of capillary-like structures. Transmission electron microscopy (TEM) analysis showed that the capillary-like structures were typical microblood vessels. ELISA demonstrated that vascularization was promoted by significant upregulation of vascularization associated growth factors due to interactions among the 3 types of cells in the bilayer, as compared to cocultures of fibroblast and endothelial cells and monocultures.
\end{abstract}

\section{Introduction}

Engineering skin substitutes hold promise for advanced treatment of acute and chronic skin wounds [1]. Skin tissue substitutes must readily adhere, have good physical and mechanical properties, and be nonantigenic [2]. Additionally, the substitutes should integrate into the host with minimal scarring and pain and facilitate angiogenesis [3]. Moreover, skin substitutes should restore functional anatomy and physiology after treatment and healing of the wound [4]. More recent skin substitutes combine epidermal and dermal layers by introducing fibroblasts and keratinocytes into an acellular matrix [5]. However, the engineering of more complex tissues consisting of large 3D structures remains a critical challenge because the penetration of oxygen, which is required for cell survival, is limited by diffusion to a distance of approximately
150 to $200 \mathrm{~mm}$ from the nearest blood vessel. Thus, the longterm survival and function of $3 \mathrm{D}$ tissues depend on the rapid development of new blood vessels to provide nutrients and oxygen to cells in the center of the tissue grafts. Sustained hypoxia or lack of nutrients leads to fibroblast dysfunction, decreased keratinocyte migration and proliferation, and tissue loss [6]. Hence, a major threat for the clinical use of dermal substitutes is insufficient vascularization leading to loosening, infection, or partial necrosis of the dermal substitute [7].

One classical approach to promoting vascularization is to decorate or supplement the scaffolds with proangiogenic factors such as VEGF, bFGF, and PDGF [8]. Different types of scaffolds containing these factors have been combined to enhance capillary formation in dermal wound healing models $[9,10]$. However, these approaches often require repeated 
administration or control release systems [11]. Skin substitutes seeded with cells producing growth factor on a continuous basis provide an attractive alternative [12]. For example, seeding fibroblasts or keratinocytes results in faster vascularization due to growth factor secretion. Previous reports have shown that VEGF overexpression in keratinocytes augments wound vascularization [13]. In vitro prevascularization can result in faster integration with the host vascular network [14]. Most of these methods involve culturing endothelial cells on or within ECM substrates (e.g., collagen, fibrin, fibronectin, laminin, etc.) or other types of biomaterials to form capillary-like structures [15]. However, these methods have some drawbacks because the ECM substitutes contained exogenous materials such as bovine collagen, dead human allogenic dermis, or synthetic polymers [16]. Scaffold choice, immunogenicity, degradation rate, toxicity of degradation products, host inflammatory responses, fibrous tissue formation due to scaffold degradation, and mechanical mismatches with the surrounding tissue are key issues that may affect the long-term behavior of the engineered tissue constructs and directly interfere with their primary biological functions [17].

In recent years, a variety of techniques have been developed to engineer tissues composed only of cells and the matrix materials that they secrete without any exogenous scaffold materials. Such techniques are even being applied for tissues where scaffolds have shown success (e.g., skin, bone, and cartilage) [18]. Scaffold-free cell sheet-based constructs have been applied for tissue repair due to their high rates of nutrient diffusion, abundant deposition of ECM, and interactions with cell membrane proteins, including growth factors, ion channels, and cell-to-cell junction proteins. Ascorbic acid has been reported to induce telomerase activity, leading to upregulation of type I collagen, fibronectin, and integrin $\beta 1$. Ascorbic acid-treated mesenchymal cells form sheets due to increased ECM production. Furthermore, Lee et al. constructed a dermal equivalent from fibroblasts treated with ascorbic acid without other exogenous materials [19]. Based on this paper, we cocultured 3 types of cells (keratinocytes, dermal fibroblasts, and dermal microvascular endothelial cells) to form scaffold-free skin equivalents containing capillary-like networks. The cocultured cells expressed high levels of vascularization-associated growth factors, including VEGF, bFGF, and PDGF, compared to cocultures of fibroblasts and endothelial cells and monocultures.

\section{Materials and Methods}

\subsection{Media Preparation}

(i) PBS (containing 10,000 U/mL penicillin and 10,000 $\mu \mathrm{g} / \mathrm{mL}$ streptomycin).

(ii) Dispase $(1.2 \mathrm{U} / \mathrm{mL})$.

(iii) Keratinocyte culture medium: K-SFM, Gibco.

(iv) Dermal fibroblast culture medium (DFM): Dulbecco's modified Eagles medium (DMEM) supplemented with $2 \%$ fetal bovine serum and $25 \mu \mathrm{g} / \mathrm{mL}$ bovine pituitary gland extract (BPE). (v) Long-term culture medium (ECM) for endothelial cells: M199 supplemented with 20\% fetal bovine serum, $25 \mu \mathrm{g} / \mathrm{mL}$ endothelial cell growth supplements (ECGS), and $90 \mu \mathrm{g} / \mathrm{mL}$ heparin.

(vi) Selective culture medium (ESM) for endothelial cells: DMEM supplemented with $10 \%$ fetal bovine serum and $150 \mu \mathrm{g} / \mathrm{mL}$ G418.

(vii) Dermis substitute culture medium (DSM): DFM with $50 \mathrm{mg} / \mathrm{mL}$ ascorbic acid.

2.2. Cell Cultures. Three types of primary cells were established from neonatal foreskin. For human epidermal keratinocytes, the foreskin was rinsed in $70 \%$ ethanol for $1 \mathrm{~min}$ and then rinsed twice in PBS. The subcutaneous fat and deep dermis were excised, and the remaining tissue was cut into small pieces. The skin pieces were then incubated in $1.2 \mathrm{U} / \mathrm{mL}$ dispase $\left(2.4 \mathrm{U} / \mathrm{mL}\right.$, Gibco BRL) at $4^{\circ} \mathrm{C}$ overnight. The tissue fragments were then transferred to a Petri dish, the epidermis and dermis were separated with fine forceps, and the epidermal sheets were transferred to a $15 \mathrm{~mL}$ centrifuge tube containing $0.25 \%$ trypsin and incubated at $37^{\circ} \mathrm{C}$ for $10 \mathrm{~min}$. Gentle pipetting was used to disaggregate the epidermal sheets into single cell suspensions. The cells were then counted and seeded at $10^{6}$ cells per two $35 \mathrm{~mm}$ dishes. Cells were incubated in DFM containing 10\% FBS for $1 \mathrm{~h}$ to facilitate attachment. The medium was then changed to KSFM. The cells were subcultured at $80 \%$ confluence. The remaining dermal parts were incubated in a preheated sterile collagenase solution $\left(625 \mathrm{U} / \mathrm{mL}\right.$, Sigma) for 2 hours at $37^{\circ} \mathrm{C}$. The dermal cells were then pipetted gently to dissociate them into a single cell suspension. Dermal fibroblasts were cultured in DFM. Endothelial cells were isolated using a Dynabeads CD31 Endothelial Cell Kit. The endothelial cells were cultured in ESM medium for 2 days to prevent fibroblasts contamination. The medium was then changed to ECM medium for long-term culture.

2.3. Flow Cytometry. The phenotypes of cultured epithelial cells, fibroblasts, and endothelial cells were identified by flow cytometry. The cultured cells were harvested using trypsin, washed twice with PBS, incubated with FITC-conjugated monoclonal antibodies (keratin 5, Factor VIII, and vimentin; all from BD Bioscience, USA), and analyzed on a FACScan. All experiments were performed at least in triplicate.

2.4. Coculture of Fibroblasts and Endothelial Cells. At passage 3 , when fibroblasts and endothelial cells reached $70-80 \%$ confluence, the cells were digested with $0.25 \%$ trypsin$0.01 \%$ EDTA for 1 minute. The reaction was quenched with $10 \mathrm{~mL}$ of bovine serum. After $5 \mathrm{~min}$ of centrifugation, the fibroblasts and endothelium were resuspended in DSM at $1.0 \times 10^{5}$ cells $/ \mathrm{mL}$. The fibroblasts and endothelial cells were cocultured on slides at ratio of $2: 1$ for more than 20 days.

2.5. Reconstruction of Scaffold-Free Bilayered Skin Containing Capillary-Like Structures. Dermal fibroblasts and endothelial cells were suspended in DSM at a concentration of 
$1.0 \times 10^{6}$ cells $/ \mathrm{mL}$ and seeded at a ratio of $2: 1$ for more than 4 weeks to produce a cell sheet. After the cell sheet was separated, four layers were superimposed and cultured for one additional week to produce a dermal equivalent. Epidermal keratinocytes were then seeded at a density of $1.0 \times$ $10^{5}$ cells $/ \mathrm{mL}$ on the dermal equivalent and cultured for one more week. Capillary-like structure localization was observed by HE staining.

2.6. Immunostaining. Cocultured cells were fixed for 30 minutes at room temperature in phosphate-buffered saline (PBS) containing $\mathrm{Ca}^{2+}, \mathrm{Mg}^{2+}$, and $4 \%$ paraformaldehyde. The fixed specimens were then transferred to $20 \%$ sucrose, frozen in optimum cutting temperature compound (Tissue Tek, Sakura, Japan), and immunostained for Factor VIII using standard protocols [20].

2.7. TEM. All protocols were according to Kunz-Shughart et al. report [21]. Bilayered engineering skin was fixed in $0.1 \mathrm{M}$ cacodylate-buffered Karnovsky's solution $(2.5 \%$ glutaraldehyde and 1\% paraformaldehyde overnight at room temperature) and postfixed in $1 \%$ osmium tetroxide $(2 \mathrm{~h})$, $\mathrm{pH} 7.3$, dehydrated in graded ethanol, and embedded in EmBed-812 epoxy resin (all reagents were obtained from Science Services, Munich, Germany; automated LYNX tissue processor, Leica, Germany). After $48 \mathrm{~h}$ heat polymerization at $60^{\circ} \mathrm{C}$, semithin $(0.8 \mu \mathrm{m})$ sections were cut, stained with toluidine blue/fuchsin, and after light microscopic spheroid selection, the epon block was trimmed for ultrathin sectioning. Ultrathin $(80 \mathrm{~nm})$ sections were prepared with a diamond knife on a Reichert Ultracut-S ultramicrotome and double contrasted with aqueous $2 \%$ uranyl acetate and lead citrate solutions for $10 \mathrm{~min}$ each. The sections were examined using a LEO912AB electron microscope operating at $80 \mathrm{kV}$.

2.8. ELISA. To compare the expression of vascularization associated growth factor under different culture conditions ((a) epithelial cell, fibroblast, and endothelial cell coculture; (b) fibroblast and endothelial cell coculture; (c) fibroblast monocultures; (d) epithelial cell monocultures; and (e) endothelial cellmonocultures), VEGF, bFGF, and PDGF expressions were measured using enzyme-linked immune sorbent assay (ELISA) kits (R\&D Systems, USA). ELISA plates were coated with monoclonal capture antibodies and blocked with bovine serum albumin $(1 \mathrm{w} / \mathrm{v} \%)$ and sucrose $(5 \mathrm{w} / \mathrm{v} \%)$ for $1 \mathrm{~h}$. Bound VEGF, bFGF, and PDGF were detected using biotin-conjugated anti-human VEGF, bFGF, and PDGF monoclonal antibodies. Streptavidin-conjugated horseradish peroxidase was added to the plates, and an enzyme substrate (tetramethylbenzidine and peroxide) was added for $20 \mathrm{~min}$. The reaction was quenched by adding an acidic solution, and absorbance was read at $450 \mathrm{~nm}$ using a PowerWave X340 plate reader (BioTEK Instruments, Inc., USA). Experiments were performed using five replicates of each sample.

2.9. Statistical Analysis. Data are expressed as mean SD. Analysis was performed using the Statistical Program for Social
Science (SPSS) 13.0 for Windows. Analysis of variance followed by Student's $t$-test was used to determine the significant differences among the groups, and $P$ values less than 0.05 were considered significant.

\section{Results}

3.1. Cell Growth and Identification. Epithelial cells isolated from neonatal foreskin displayed typical cobblestone morphology until at least passage 10 (Figure 1(a)). At passage 3, 92\% of epithelial cells were keratin 5 positive as determined by flow cytometry (Figure 1(b)). Dermal fibroblasts displayed bipolar spindle morphology until at least passage 20 (Figure $1(\mathrm{c})$ ). At passage 3, 98\% of dermal cells were vimentin-posi-tive by flow cytometry (Figure 1(d)).

Endothelial cells have a characteristic appearance that distinguishes them from other cell types. At low density, primary endothelial cells are elongated. However, when $70-$ $80 \%$ confluence is reached (or after subculture), endothelial cells display a characteristic cobblestone morphology for up to 15 passages (Figure 1(e)). Primary endothelial cultures were subcultured when cells reached $70-80 \%$ confluence. After 2 days in culture, fibroblast contamination was observed in some areas. However, after culture in ESM for another 3 days, fibroblasts contamination was eliminated. Endothelia cells can be maintained in ECM for a long period of time without obvious changes in cell morphology or proliferation. Up to 95\% of endothelial cells were Factor VIII-positive by flow cytometry (Figure 1(f)).

3.2. Cocultures of Endothelial Cells and Fibroblasts. When endothelial cells and fibroblasts were cocultured, the fibroblasts proliferated and synthesized new matrix materials. At 20 days, abundant matrix secretion was observed. Lumenlike structures were also observed by light microscopy (Figure 2(a)). Moreover, factor VIII immunofluorescence showed that the lumen-like structures were formed by endothelial cells and distributed throughout the construct. This result suggests that dermal fibroblast specific matrix proteins support endothelia cell migration and the formation of a capillary-like tubular structure (Figure 2(b)).

\subsection{Reconstruction of the Skin Equivalent Containing Capil-} lary-Like Structures. After 5 weeks of fibroblast-endothelium coculture, epithelial cells were seeded on the constructs to form bilayered skin. One week after-seeding, the skin was harvested from the culture dish. The skin equivalents were approximately $1 \mathrm{~mm}$. HE staining showed that the skin exhibited a stratified epidermis composed of a cuboidal basal layer, suprabasal layers, a granular layer expressing filaggrin and transglutaminase, and a stratum corneum (Figure 2(c)). Moreover, Factor VIII immunostaining revealed that interactions between epithelial cells and fibroblast enhanced capillary-like network formation, as compared to cocultures of endothelial cells and fibroblasts (Figure 2(d)). To verify that the capillary-like network was composed of microblood vessels, TEM was used to visualize the ultrastructure of 


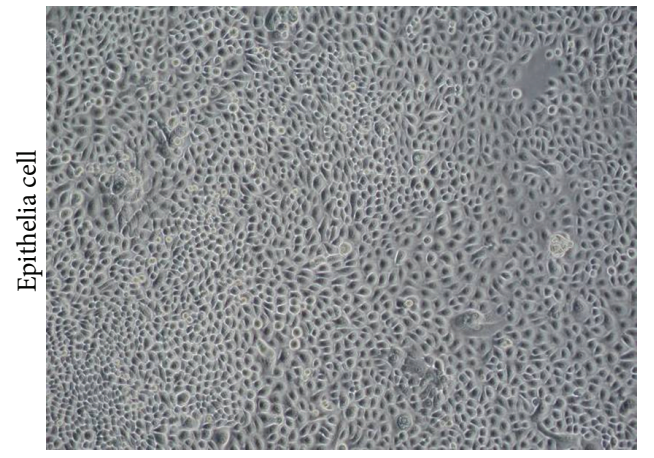

(a)

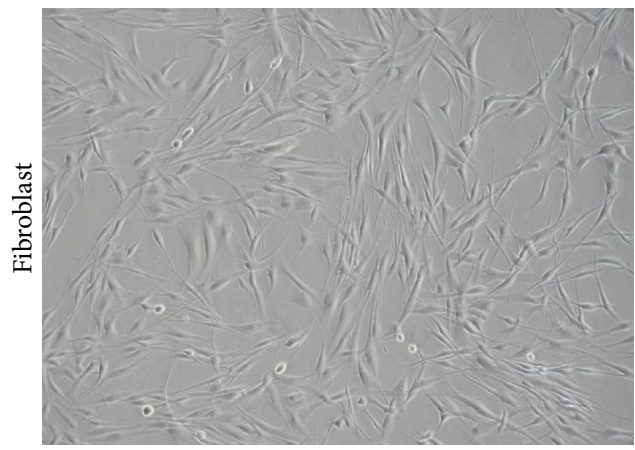

(c)

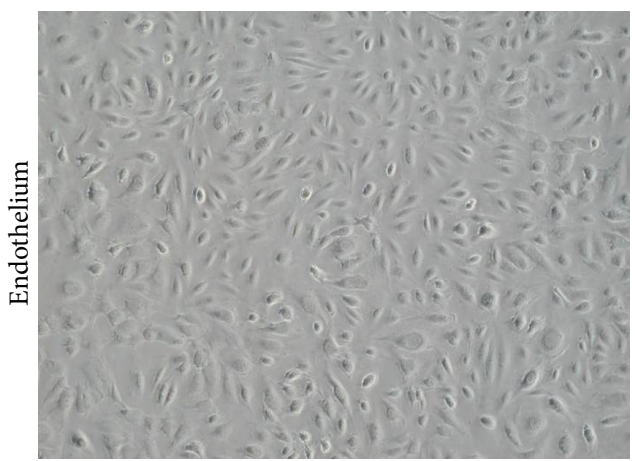

(e)

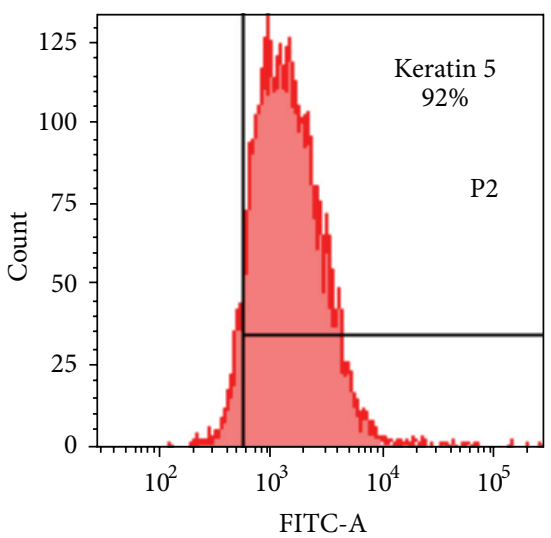

(b)

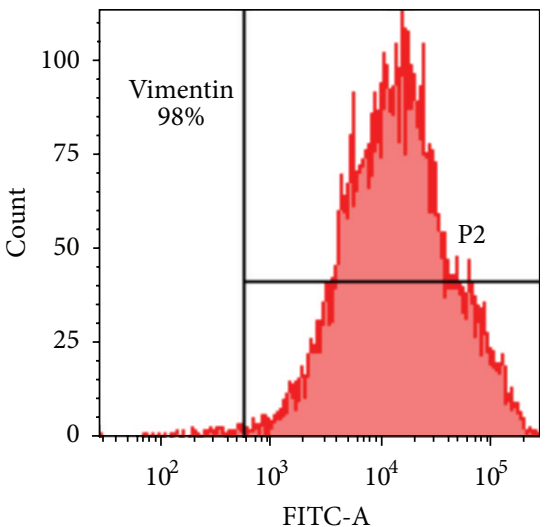

(d)

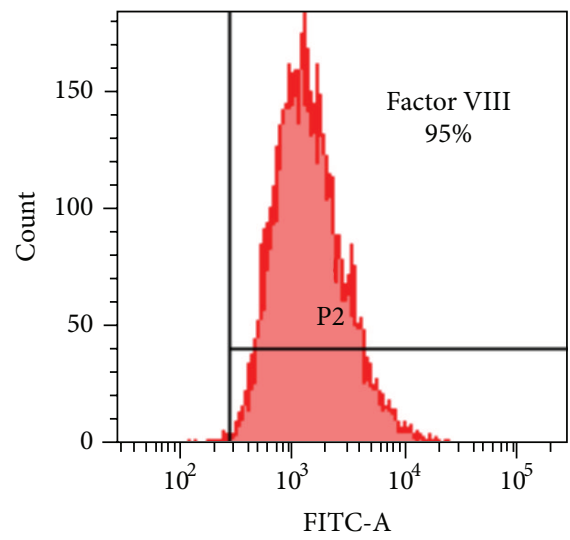

(f)

Figure 1: (a) Morphological observation of epithelium culture on Petri dish by light microscope at passage 3. (b) Keratin 5 flow cytometry analysis; the positive rate reached to $92 \%$. (c) Morphological observation of fibroblast culture on Petri dish by light microscope at passage 3. (d) Vimentin flow cytometry analysis; the positive rate reached to 98\%. (e) Morphological observation of endothelium culture on Petri Dish by light microscope at passage 3. (f) Factor VIII flow cytometry analysis; the positive rate reached to 95\%.

lumens in the tissue-engineered skin. The endothelium component of the dermis formed typical micro-blood vessel structures (Figures $2(\mathrm{e})$ and 2(f)).

3.4. Epithelial Cells Promote New Blood Vessel Formation in Tissue-Engineered Skin. Capillaries were nearly 3-fold more abundant in bilayered skin than in dermis (Figure 3(a)).
Interactions between epithelial cells and fibroblasts regulate growth factor expression [22]; therefore, ELISA was used to compare the expression of vascularization-associated growth factors across the 5 groups. Expressions of VEGF, bFGF, and PDGF were upregulated 6-, 3-, and 4-fold, respectively, in epithelial cell, fibroblast, and endothelial cell cocultures compare to fibroblast and endothelial cell co-cultures 


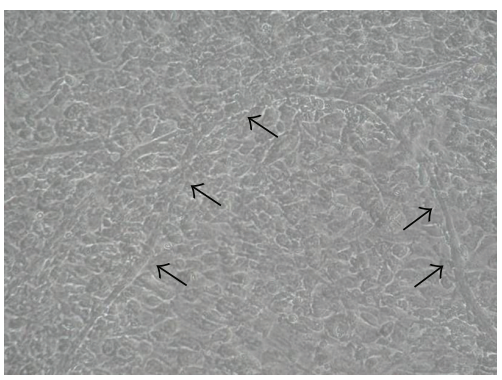

(a)

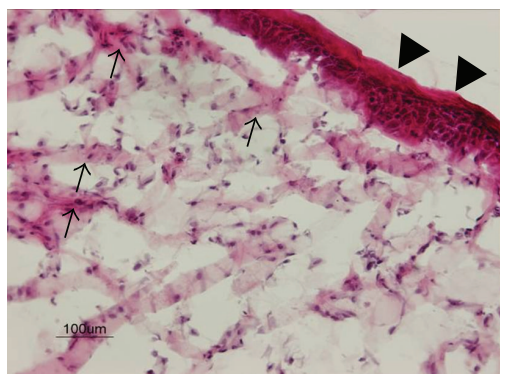

(c)

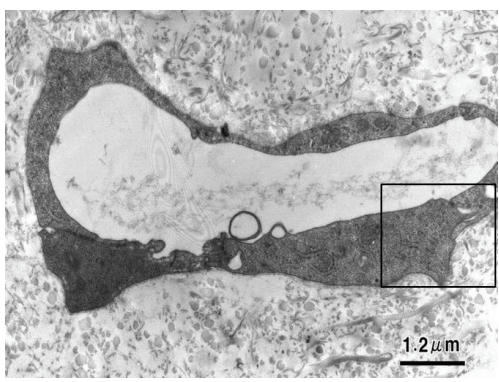

(e)

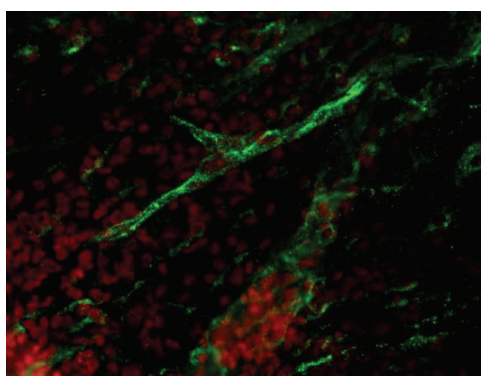

(b)

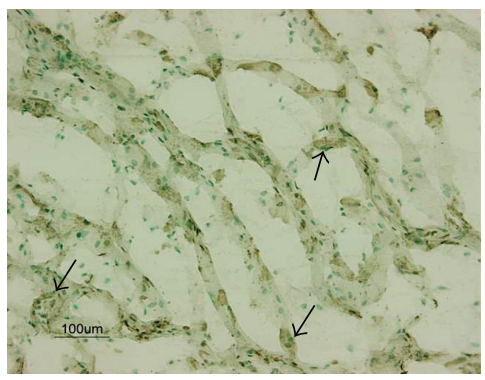

(d)

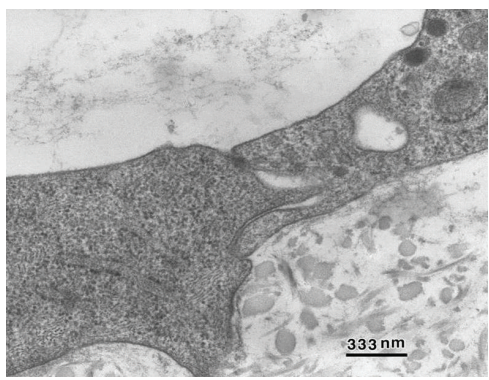

(f)

Figure 2: (a) The morphological observation of coculture of fibroblast and endothelium after 20 days. Abundant matrix secretion and lumenlike structures could be observed by light microscopy. Arrows point to the lumen-like structure. (b) Factor VIII immunofluorescence showed that the lumen-like structures were formed by endothelial cells and distributed throughout the construct. Green was the Factor VIII positive staining, and red was the cell nuclei. (c) HE staining of bilayered tissue-engineered skin. HE staining showed that the skin exhibited a stratified epidermis composed of a cuboidal basal layer, suprabasal layers, a granular layer expressing filaggrin and transglutaminase, and a stratum corneum. In dermis, a large number of cells gathered in the collagen fiber. Triangles point to the epidermis, and arrows point to the dermis. (d) Factor VIII immunostaining of bilayered tissue-engineered skin. Dark brown stain was positive for factor VIII. Results showed a large number of factor VIII expressed endothelium distributed in dermis forming lumen-like structure. Arrows point to the lumen-like structure. ((e) and (f)) TEM observation of ultrastructure of capillary in bilayered tissue-engineered skin. The morphology showed that capillary had a typical micro-blood vessel structure.

(Figures 3(b), 3(c), and 3(d)). VEGF expression was not significantly different between fibroblast monocultures and co-cultures of fibroblast and endothelial cells; however, VEGF expression was significantly higher in fibroblast monocultures compared to epithelial cell and endothelial cell monocultures (Figure 3(b)). PDGF expression followed a similar trend to VEGF expression (Figure 3(d)). bFGF expression was significantly lower in endothelial cell monocultures compared to the other groups, although the differences among the remaining 3 groups were not significant (Figure 3(c)).

\section{Discussion}

Tissue engineering seeks to replace damaged, injured, or missing tissues with biologically compatible substitutes [23].
Tissue-engineering substitutes offer many advantages over traditional therapies [24]. However, the engineering of more complex tissues consisting of large 3D structures is challenging due to limitations in vascularization and angiogenesis. Oxygen and nutrients required for cell survival become limiting at approximately 150 to $200 \mathrm{~mm}$ from blood vessel; therefore, cells far from the wound surface die from hypoxia or lack of nutrients [25]. Accordingly, tissue-engineering research has focused on understanding the formation of new blood vessels. Skin substitutes formed in vitro with vascular networks inosculate faster with the host vascular network [26]. This study describes an approach to construct scaffold-free bilayered skin containing capillary networks by epithelial cells, fibroblasts, and endothelial cells. In our system, epithelial cells and cocultured fibroblasts secrete 


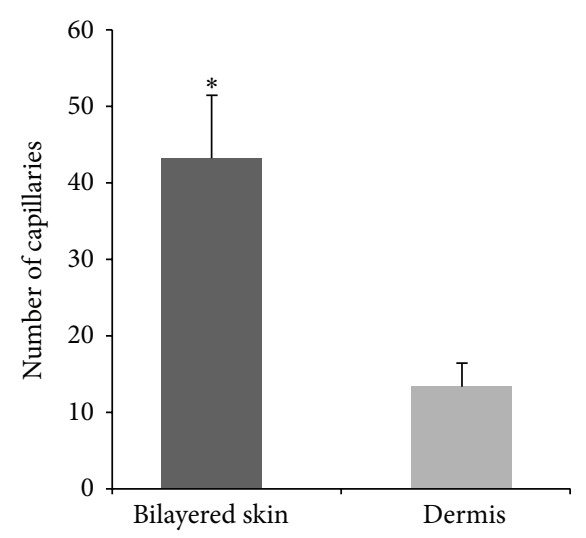

(a)

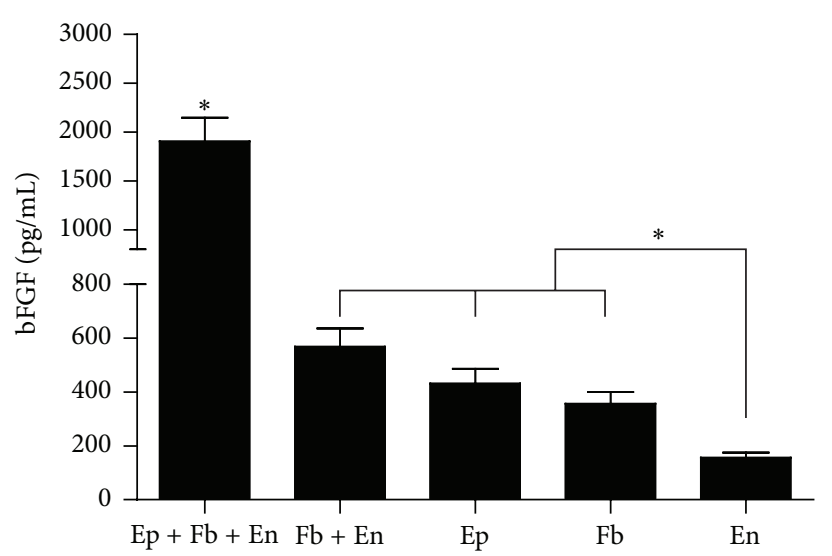

(c)

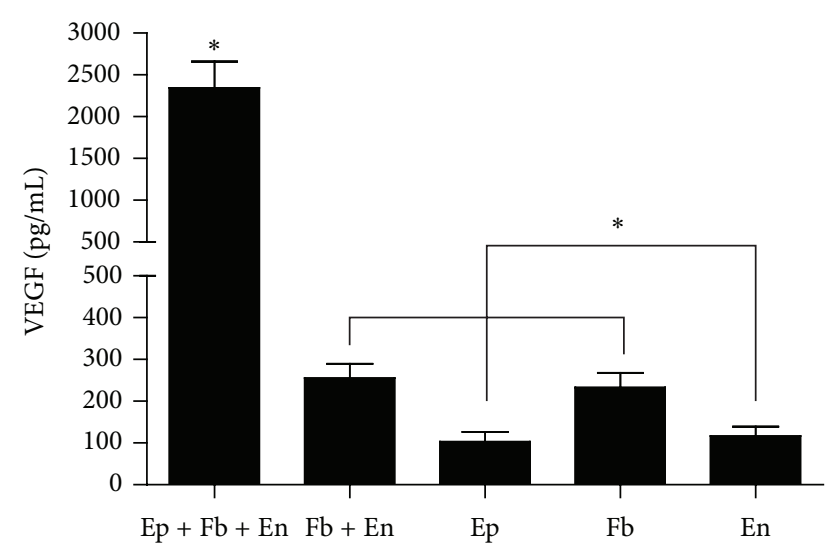

(b)

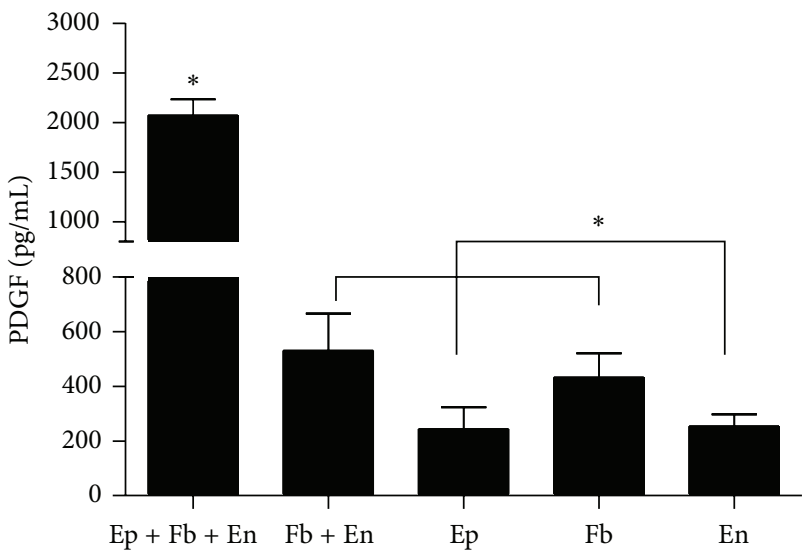

(d)

FIGURE 3: (a) The comparison of capillary network between bilayered engineering skin and dermis by image analysis. (b) The comparison of VEGF expression during different culture conditions by ELISA analysis. (c) The comparison of bFGF expression during different culture conditions by ELISA analysis. (d) The comparison of PDGF expression during different culture conditions by ELISA analysis.

specific matrix components that stimulate endothelial cells to form capillary networks in the dermis. We found that crosstalk among these 3 cell types upregulates the expression of vascularization associated growth factors, including VEGF, bFGF, and PDGF.

Endothelial cell co-cultures represent a starting point for vascularization [27]. Endothelial cell spheroids produce capillary-like sprouts, especially in the presence of fibroblasts. Fibroblasts modulate endothelial cell network formation, suggesting a critical need for complex mixed spheroid cocultures to adequately mimic in vivo angiogenesis [21]. Fibroblasts are also a rich source of growth factors for self-stimulation and for activation of other cell types. Activated fibroblasts produce angiogenic growth factors, such as VEGF, bFGF, and PDGF [28]. Seeding only fibroblast in a scaffold can thus promote vascularization due to growth factor secretion [12]. We obtained similar results when cocultured dermal fibroblasts caused hDMVECs to migrate and form capillarylike tubular structures in the fibroblast-derived matrix. Furthermore, capillary formation was enhanced by seeding epithelial cells due to the upregulation of vascularization associated growth factors. These results were not improved by increasing the number of cells, suggesting that cross-talk occurred among epithelial cells, fibroblasts, and endothelial cells. Our ELISA results demonstrate that VEGF, bFGF, and PDGF are also upregulated in epithelial cell cocultures compared to cultures without epithelial cells containing the same number of cells. The interactions between epithelial cells and fibroblasts are intimately involved in a number of functions within the skin, including tissue differentiation during skin development, responses to and regulation of local inflammatory factors, and repair of damaged tissue [29]. Thus, these interactions mimic skin development in vivo, enhancing the formation of 3D vessel-like structures.

Extracellular matrix components also play important roles in blood vessel formation [30-32]. Endothelial cells require adhesion molecules and growth factors to promote their growth and tubular organization. In vitro angiogenesis models usually combine endothelial cells with various purified extracellular matrix components, such as collagen [33], fibrin [34], and fibrinogen [35], or multimolecular matrices such as Matrigel [36]. Thus, exogenous ECM is usually applied to form capillary-like structures. However, these exogenous biomaterials may impart unforeseeable cellbiomaterial interactions, uneven degradation, inflammatory responses, and infection, or may limit cell adhesion [37]. To 
avoid these disadvantages, we prepared bilayered tissueengineered skin without any exogenous biomaterials. The matrix components of the dermis were secreted by fibroblasts. Fibroblast-derived matrix proteins, such as collagen 1, PCOLCE, SPARC, IGFBP7, and $\beta$ ig-h3, induce endothelial cell sprouting and are necessary for lumen formation [38]. In addition, dermis-specific matrix components secreted by fibroblasts deposit numerous growth factors and adhesion molecules that can promote blood vessel formation and skin repair $[39,40]$. Therefore, tissue-engineered skin has a strong capacity for forming capillary networks.

\section{Conclusion}

We described an approach to construct scaffold-free bilayered skin containing capillary networks from epithelial cells, fibroblasts, and endothelial cells. We first prepared a dermal sheet from fibroblasts and endothelial cells. After 20 days in culture, epithelial cells were seeded on the scaffold-free dermis to form bilayered skin. Capillaries were 3-fold more abundant in bilayered skin than in dermis. The capillarylike networks contained typical micro-blood vessels. ELISA showed that interactions among the 3 cell types upregulated vascularization-associated growth factor expression compared to cocultures of fibroblast and endothelial cells and monocultures.

\section{Authors' Contribution}

Y. Liu and H. Luo contributed equally to this paper.

\section{Conflict of Interests}

All the authors of this study do not have any direct financial relationship with commercial identities.

\section{Acknowledgments}

This study was supported by the funding support from National High Technology Research and Development Program of China (Grant no. 2012AA020507).

\section{References}

[1] M. Chen, M. Przyborowski, and F. Berthiaume, "Stem cells for skin tissue engineering and wound healing," Critical Reviews in Biomedical Engineering, vol. 37, no. 4-5, pp. 399-421, 2009.

[2] S. MacNeil, "Progress and opportunities for tissue-engineered skin," Nature, vol. 445, no. 7130, pp. 874-880, 2007.

[3] B. Hendrickx, J. J. Vranckx, and A. Luttun, "Cell-based vascularization strategies for skin tissue engineering," Tissue Engineering Part B, vol. 17, no. 1, pp. 13-24, 2011.

[4] A. D. Metcalfe and M. W. J. Ferguson, “Tissue engineering of replacement skin: the crossroads of biomaterials, wound healing, embryonic development, stem cells and regeneration," Journal of the Royal Society Interface, vol. 4, no. 14, pp. 413-417, 2007.

[5] D. M. Supp and S. T. Boyce, "Engineered skin substitutes: practices and potentials," Clinics in Dermatology, vol. 23, no. 4, pp. 403-412, 2005.
[6] A. Siddiqui, R. D. Galiano, D. Connors, E. Gruskin, L. Wu, and T. A. Mustoe, "Differential effects of oxygen on human dermal fibroblasts: acute versus chronic hypoxia," Wound Repair and Regeneration, vol. 4, no. 2, pp. 211-218, 1996.

[7] P. S. Sahota, J. L. Burn, M. Heaton et al., "Development of a reconstructed human skin model for angiogenesis," Wound Repair and Regeneration, vol. 11, no. 4, pp. 275-284, 2003.

[8] A. H. Zisch, M. P. Lutolf, and J. A. Hubbell, "Biopolymeric delivery matrices for angiogenic growth factors," Cardiovascular Pathology, vol. 12, no. 6, pp. 295-310, 2003.

[9] A. H. Zisch, M. P. Lutolf, M. Ehrbar et al., "Cell-demanded release of VEGF from synthetic, biointeractive cell ingrowth matrices for vascularized tissue growth," FASEB Journal, vol. 17, no. 15, pp. 2260-2262, 2003.

[10] J. Andrae, R. Gallini, and C. Betsholtz, "Role of platelet-derived growth factors in physiology and medicine," Genes and Development, vol. 22, no. 10, pp. 1276-1312, 2008.

[11] T. Kitajima, H. Terai, and Y. Ito, "A fusion protein of hepatocyte growth factor for immobilization to collagen," Biomaterials, vol. 28, no. 11, pp. 1989-1997, 2007.

[12] S. W. Spiekstra, M. Breetveld, T. Rustemeyer, R. J. Scheper, and S. Gibbs, "Wound-healing factors secreted by epidermal keratinocytes and dermal fibroblasts in skin substitutes," Wound Repair and Regeneration, vol. 15, no. 5, pp. 708-717, 2007.

[13] S. Dickens, P. Vermeulen, B. Hendrickx, S. Van Den Berge, and J. J. Vranckx, "Regulable vascular endothelial growth factor165 overexpression by ex vivo expanded keratinocyte cultures promotes matrix formation, angiogenesis, and healing in porcine full-thickness wounds," Tissue Engineering Part A, vol. 14, no. 1, pp. 19-27, 2008.

[14] P. S. Sahota, J. L. Burn, N. J. Brown, and S. MacNeil, “Approaches to improve angiogenesis in tissue-engineered skin," Wound Repair and Regeneration, vol. 12, no. 6, pp. 635-642, 2004.

[15] M. Lovett, K. Lee, A. Edwards, and D. L. Kaplan, "Vascularization strategies for tissue engineering," Tissue Engineering Part B, vol. 15, no. 3, pp. 353-370, 2009.

[16] S. Chaterji, K. Park, and A. Panitch, "Scaffold-free in vitro arterial mimetics: the importance of smooth muscle-endothelium contact," Tissue Engineering Part A, vol. 16, no. 6, pp. 1901-1912, 2010.

[17] D. F. Williams, "On the mechanisms of biocompatibility," Biomaterials, vol. 29, no. 20, pp. 2941-2953, 2008.

[18] J. M. Kelm and M. Fussenegger, "Scaffold-free cell delivery for use in regenerative medicine," Advanced Drug Delivery Reviews, vol. 62, no. 7-8, pp. 753-764, 2010.

[19] D. Y. Lee, J. H. Lee, J. M. Yang, E. S. Lee, K. H. Park, and G. H. Mun, "A new dermal equivalent: the use of dermal fibroblast culture alone without exogenous materials," Journal of Dermatological Science, vol. 43, no. 2, pp. 95-104, 2006.

[20] B. S. Yoon, S. J. Yoo, J. E. Lee, S. You, H. T. Lee, and H. S. Yoon, "Enhanced differentiation of human embryonic stem cells into cardiomyocytes by combining hanging drop culture and 5-azacytidine treatment," Differentiation, vol. 74, no. 4, pp. 149-159, 2006.

[21] L. A. Kunz-Schughart, J. A. Schroeder, M. Wondrak et al., "Potential of fibroblasts to regulate the formation of threedimensional vessel-like structures from endothelial cells in vitro," American Journal of Physiology, vol. 290, no. 5, pp. C1385C1398, 2006.

[22] D. Knight, "Epithelium-fibroblast interactions in response to airway inflammation," Immunology and Cell Biology, vol. 79, no. 2, pp. 160-164, 2001. 
[23] M. Nomi, A. Atala, P. De Coppi, and S. Soker, "Principals of neovascularization for tissue engineering," Molecular Aspects of Medicine, vol. 23, no. 6, pp. 463-483, 2002.

[24] A. Gaspar, L. Moldovan, D. Constantin, A. M. Stanciuc, P. M. Sarbu Boeti, and I. C. Efrimescu, "Collagen-based scaffolds for skin tissue engineering," Journal of Medicine and Life, vol. 4, no. 2, pp. 172-177, 2011.

[25] M. W. Laschke, Y. Harder, M. Amon et al., "Angiogenesis in tissue engineering: breathing life into constructed tissue substitutes," Tissue Engineering, vol. 12, no. 8, pp. 2093-2104, 2006.

[26] P. L. Tremblay, V. Hudon, F. Berthod, L. Germain, and F. A. Auger, "Inosculation of tissue-engineered capillaries with the host's vasculature in a reconstructed skin transplanted on mice," American Journal of Transplantation, vol. 5, no. 5, pp. 1002-1010, 2005.

[27] A. Wenger, N. Kowalewski, A. Stahl et al., "Development and characterization of a spheroidal coculture model of endothelial cells and fibroblasts for improving angiogenesis in tissue engineering," Cells Tissues Organs, vol. 181, no. 2, pp. 80-88, 2006.

[28] D. Ruiter, T. Bogenrieder, D. Elder, and M. Herlyn, "Melanomastroma interactions: structural and functional aspects," The Lancet Oncology, vol. 3, no. 1, pp. 35-43, 2002.

[29] L. P. Kamolz, M. Luegmair, N. Wick et al., "The Viennese culture method: cultured human epithelium obtained on a dermal matrix based on fibroblast containing fibrin glue gels," Burns, vol. 31, no. 1, pp. 25-29, 2005.

[30] W. Friess, "Collagen-biomaterial for drug delivery," European Journal of Pharmaceutics and Biopharmaceutics, vol. 45, no. 2, pp. 113-136, 1998.

[31] P. M. Royce, T. Kato, K. I. Ohsaki, and A. Miura, "The enhancement of cellular infiltration and vascularisation of a collagenous dermal implant in the rat by platelet-derived growth factor BB," Journal of Dermatological Science, vol. 10, no. 1, pp. 42-52, 1995.

[32] Y. M. Elçin, V. Dixit, and G. Gitnick, "Extensive in vivo angiogenesis following controlled release of human vascular endothelial cell growth factor: implications for tissue engineering and wound healing," Artificial Organs, vol. 25, no. 7, pp. 558565, 2001.

[33] R. K. Schneider, J. Anraths, R. Kramann et al., "The role of biomaterials in the direction of mesenchymal stem cell properties and extracellular matrix remodelling in dermal tissue engineering," Biomaterials, vol. 31, no. 31, pp. 7948-7959, 2010.

[34] D. G. Chalupowicz, Z. A. Chowdhury, T. L. Bach, C. Barsigian, and J. Martinez, "Fibrin II induces endothelial cell capillary tube formation," Journal of Cell Biology, vol. 130, no. 1, pp. 207-215, 1995.

[35] D. E. Ingber and J. Folkman, "Mechanochemical switching between growth and differentiation during fibroblast growth factor-stimulated angiogenesis in vitro: role of extracellular matrix," Journal of Cell Biology, vol. 109, no. 1, pp. 317-330, 1989.

[36] Y. Kubota, H. K. Kleinman, G. R. Martin, and T. J. Lawley, "Role of laminin and basement membrane in the morphological differentiation of human endothelial cells into capillary-like structures," Journal of Cell Biology, vol. 107, no. 4, pp. 1589-1598, 1988.

[37] C. Norotte, F. S. Marga, L. E. Niklason, and G. Forgacs, "Scaffold-free vascular tissue engineering using bioprinting," Biomaterials, vol. 30, no. 30, pp. 5910-5917, 2009.

[38] A. C. Newman, M. N. Nakatsu, W. Chou, P. D. Gershon, and C. C. Hughes, "The requirement for fibroblasts in angiogenesis: fibroblast-derived matrix proteins are essential for endothelial cell lumen formation," Molecular Biology of the Cell, vol. 22, no. 20, pp. 3791-3800, 2011.

[39] G. Jin, M. P. Prabhakaran, and S. Ramakrishna, "Stem cell differentiation to epidermal lineages on electrospun nanofibrous substrates for skin tissue engineering," Acta Biomaterialia, vol. 7, no. 8, pp. 3113-3122, 2011.

[40] G. Galassi, P. Brun, M. Radice et al., "In vitro reconstructed dermis implanted in human wounds: degradation studies of the HA-based supporting scaffold," Biomaterials, vol. 21, no. 21, pp. 2183-2191, 2000. 


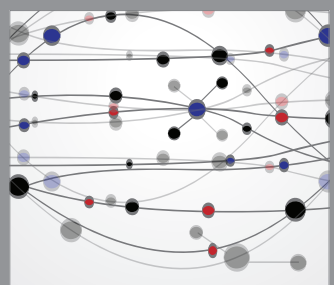

The Scientific World Journal
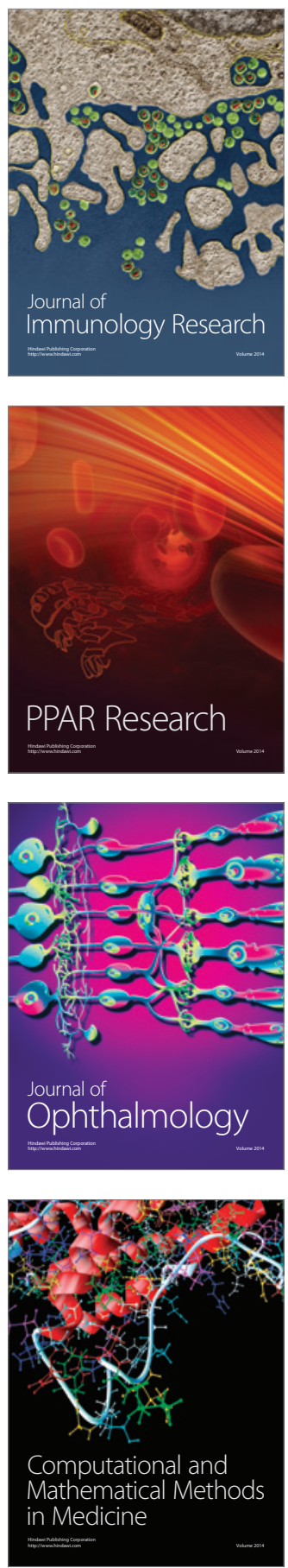

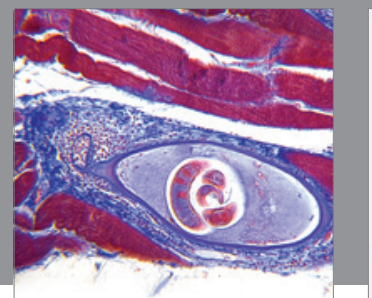

Gastroenterology

Research and Practice
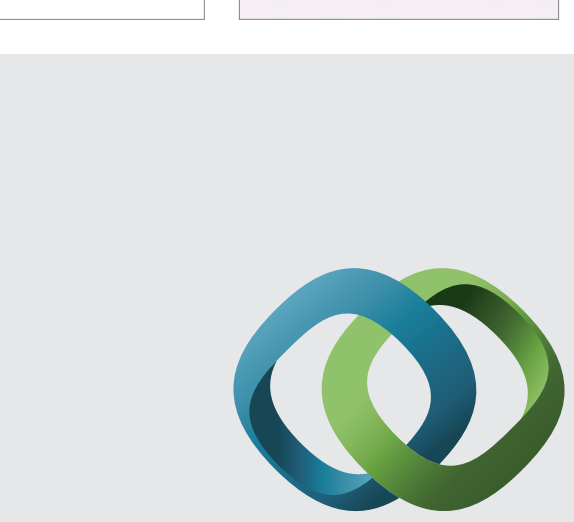

\section{Hindawi}

Submit your manuscripts at

http://www.hindawi.com
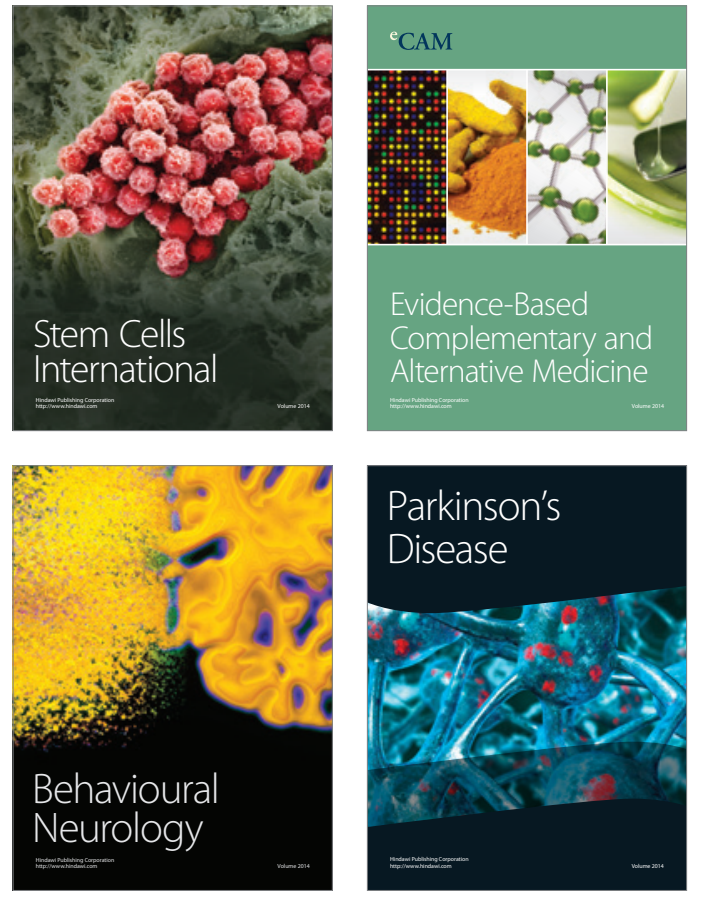
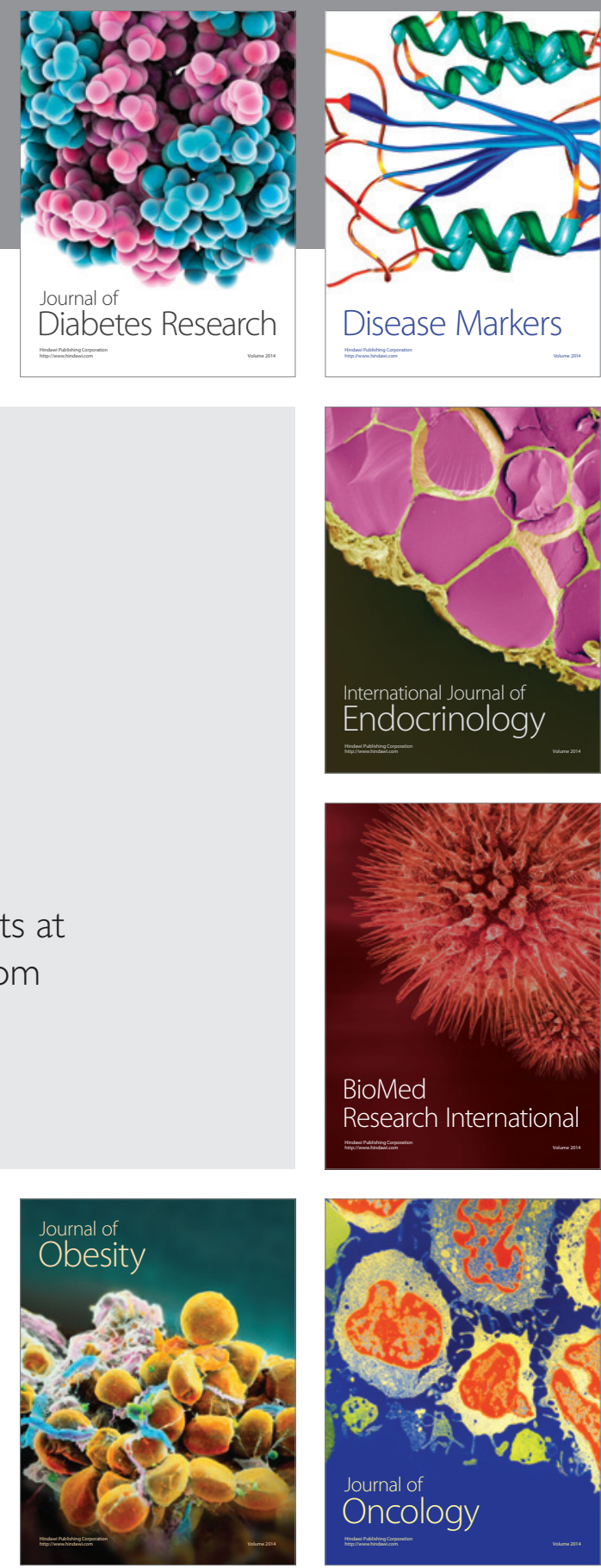

Disease Markers
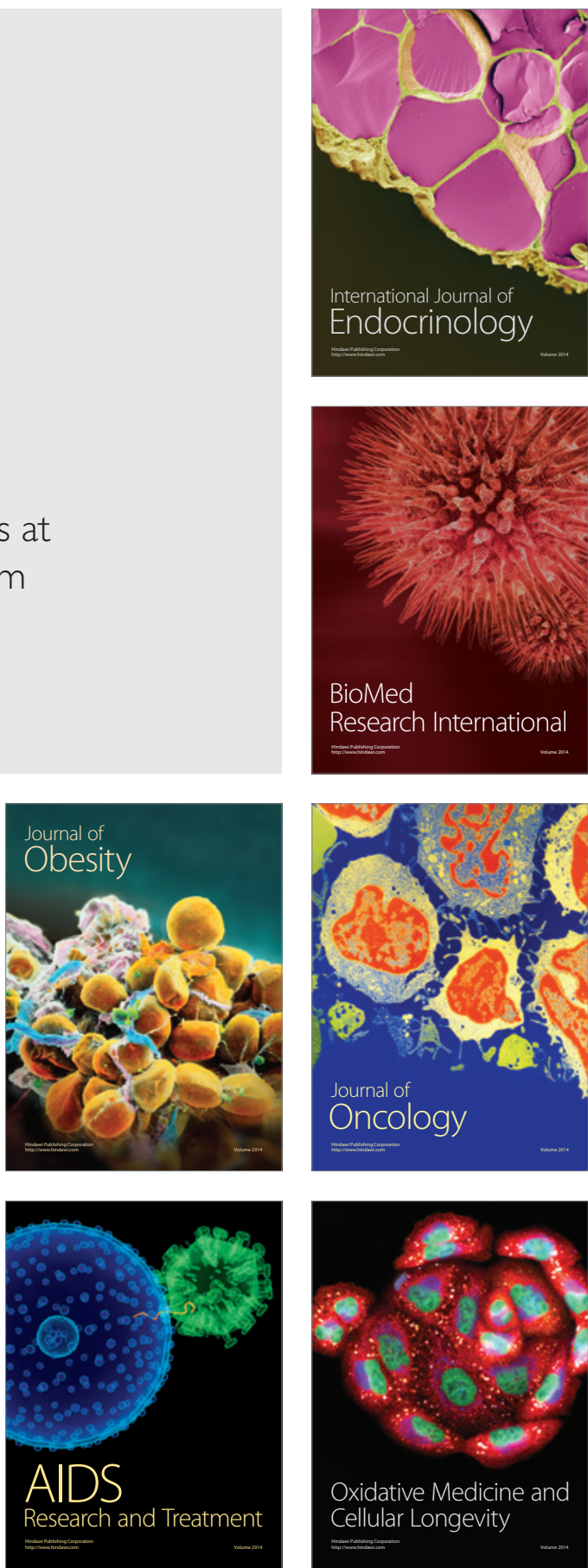